\title{
Human Immunodeficiency Virus: Discovery to Drug Resistance - A Review Update
}

\author{
Arpan Acharya ${ }^{1}$, Salil Vaniawala ${ }^{1}$, Harsh Parekh ${ }^{2}$, Rabindra Nath Misra ${ }^{3}$, Minal Wani ${ }^{4}$ and Pratap N Mukhopadhyaya ${ }^{{ }^{*}}$ \\ ${ }^{1}$ SN Genelab, 2nd Floor, President Plaza, Tower A, Nanpura, Ring Road, Surat, Gujarat, India \\ 2Department of Biotechnology, JJT University, Churu Jhunjhunu Road, Vidyanagari,, Chudela, Rajasthan, India \\ ${ }^{3}$ Department of Microbiology, Padmashree Dr D Y Patil Medical College \& Research Centre, Sant Tukaram Nagar, Pimpri, Pune - 411 018, Maharashtra, India \\ ${ }^{4}$ Dr. D. Y. Patil Biotechnology and Bioinformatics Institute, Tathawade, Pune, India
}

*Corresponding author: Mukhopadhyaya PN, B-604, Kapil Aasmant, Pashan-Sus Road, Pashan, Pune-411 021, Maharashtra, India, Tel: +91 98811 53425; E-mail: pnm.correspondingauthor@yahoo.com

Received date: Dec 02, 2015; Accepted date: Jan 07, 2016; Published date: Jan 15, 2016

Copyright: () 2016 Acharya A, et al. This is an open-access article distributed under the terms of the Creative Commons Attribution License, which permits unrestricted use, distribution, and reproduction in any medium, provided the original author and source are credited.

\begin{abstract}
Since year 1983 when Human Immunodeficiency virus (HIV) was first discovered, there has been a spectacular progress in understanding the complex biology of the virus. Drug discovery accepted the challenge to stem spread of pathogenic virus that was characterized by unparalleled ability to alter, survive and kill humans. The genetic diversity of this virus, attributed to an extraordinary rate of mutation of the genome, is now the center of most of the scientific hurdles met towards its prevention and control. While the disease caused by HIV was staged by the medical community, a range of drugs were almost simultaneously discovered to address it. This was followed by the challenge of managing the menace of HIV drug resistance. The unique mechanism of host invasion and reduction in immune strength of the victim opened an additional area of concern by way of secondary infection which is now the prominent immediate cause of death of patients. In this review we take a panoramic view of this entire scenario and discuss the need for private sector to contribute to research and development in order to keep pace with the complexity of the virus and bring about a sustained solution to the alarming disease caused by it.
\end{abstract}

Keywords: Human immunodeficiency virus; Classification; Genome; Drugs; Drug resistance

\section{Background}

\section{Discovery of human immunodeficiency virus (HIV) and} AIDS

In the year 1981 Center for Disease Control (CDC) in United States of America reported an outbreak of Kaposi's Sarcoma (KS) among homosexuals, in general which is considered to be a less sever benign cancer [1]. In the same year Gottlieb et al. reported another outbreak of Pneumocystis Carinii Pneumonia (PCP) in the city of New York among homosexuals. These two incidences were considered as the first reported incidence of Acquired Immunodeficiency Syndrome (AIDS). This was followed by sudden increase in opportunistic infections among gay men and Hemophilic patients [2].

In the year 1983, a group of researchers from Pasteur Institute of France isolated a new virus and suggest that it may be the agent responsible for AIDS. The virus was named as lymphadenopathyassociated virus (LAV) [3]. As the scientific community trying discovers the root cause of AIDS, the disease started to spread among women and Childs having no other risk factors [4].

In the year 1984, a group of scientist from National Cancer Institute (NCI, USA) claimed to discover a new virus called human Tlymphotropic virus type III (HTLV-III) to be the agent responsible for AIDS [5].
In the year 1985, it was confirmed that LAV and HTLV-III were same virus [6]. In the same year US-FDA approved commercial production of a blood test to detect the presence of antibodies to LAV/ HTLV-III. As the controversy over the initial discoverer of AIDS virus remained unsolved, in the year 1986 the International Committee on Taxonomy of Viruses, dropped previous two names of AIDS virus and named it as Human Immunodeficiency Virus (HIV) [7].

\section{HIV virology: structure and genomic organization}

Human Immunodeficiency Virus is a retrovirus. It is spherical in shape and its diameter is around 110 nanometer. The envelope of HIV is composed of lipid bi-layer which is derived from the host cell membrane during the budding of new virions. As HIV envelop generated from host cell membrane, a number of host cell membrane proteins remain entrapped within it. HIV envelop also have two glycoprotein's gp41 and gp120, composed of sugar and protein molecules. gp120 helps in anchoring of viral particles with CD4 receptors of T-helper cells, macrophages and monocytes. gp41 helps in fusion of viral particles with host cell membrane and entry of viral core within the cell [8].

The core of HIV is cone shaped and composed of nucleocapsids. It has two layers, the outer layer is composed of p17 matrix protein and the inner layer is composed of p24 protein. p17 participates in viral replication and helps the entry of envelop in virion during formation of new viruses [9]. p24 is the most abundant protein within the core of HIV particles. It helps in the transportation of viral genetic materials to host cell nucleolus after entry and reverse transcription. Inside the core, at the center of viral particle two copies of single stranded RNA molecules constitute HIV genome. The genetic material remains 
bounded by nucleocapsid protein $\mathrm{p} 7$. p6 protein plays an important role in viral budding [10]. The three viral enzymes reverse transcriptase, integrase and protease also remain inside the core of HIV (Figure 1).

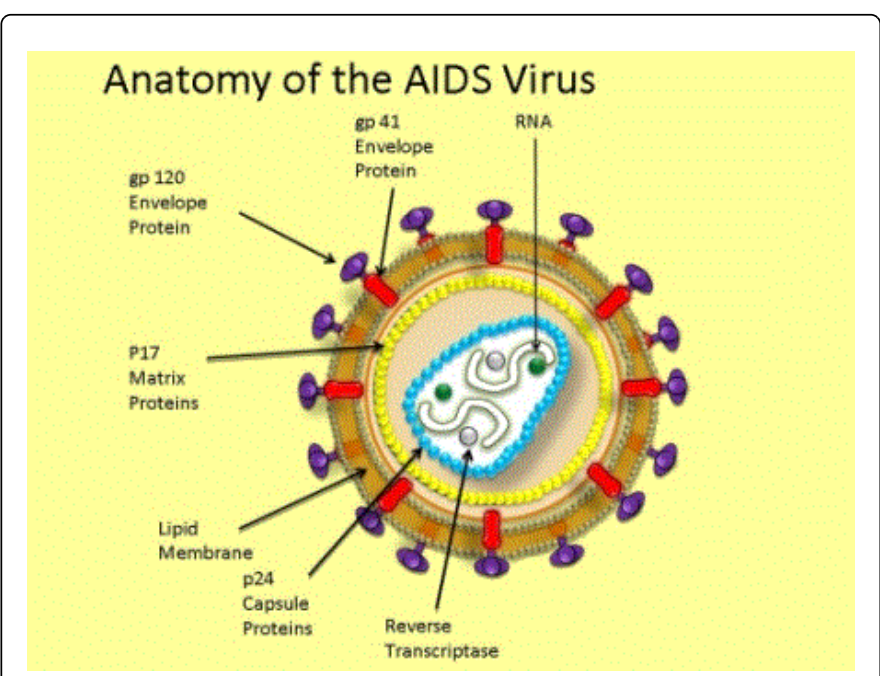

Figure 1: Structure of human immunodeficiency virus (HIV).

Reverse Transcriptase converts single stranded RNA molecule of HIV into double stranded DNA, which is integrated in the host cell genome with the aid of integrase enzyme. HIV RT also has a ribonuclease activity, which helps in digestion of RNA in the RNADNA hybrid and generates single stranded DNA. This acts as a template during formation of double stranded DNA. The main function of HIV protease is to cleave the long polypeptides synthesized from the different ORF of HIV genome integrated in the host genome to generate functional proteins.

HIV genetic material remains in two forms, as two copies of single stranded RNA inside the intact viral particles and as a double stranded DNA integrated in the host genome. This double stranded DNA also known as proviral DNA. The size of HIV genome is around $9.7 \mathrm{~KB}$. The proviral DNA have two long terminal repeats (LTR) in the 5' and 3 ' end of HIV open reading frame. This LTR's actively take part in integration of viral genome in host chromosome and also acts as a promoter during transcription of proviral DNA.

HIV genome has 9 overlapping ORF which codes for 15 proteins (Figure 2). The Gag-Pol-Env ORF codes for viral enzymes, structural proteins and envelop glycoproteins. Three regulatory proteins of HIV transcribed by Tat, Rav and Nef ORF and three accessory proteins are generated from Vif, Vpr and Vpu ORF respectively. The Gag-Pol ORF initially transcribed as a $160 \mathrm{KDaGag}$-Pol precursor, which is then auto catalyzed to form p55Gag and three viral enzymes reverse transcriptase (RT), protease (PR) and integrase (IN) respectively. $\mathrm{p} 55 \mathrm{Gag}$ is cleaved by viral protease to form functional matrix protein p17, capsid protein p24 and nuleocapsid proteins p6 and p7 respectively. The Env ORF also initially transcribed as a $160 \mathrm{KDa}$ precursor, which with the help of host cell enzymes cleaved and generates gp41 and gp120 glycoproteins. The long unprocessed viral polypeptides are non-functional.

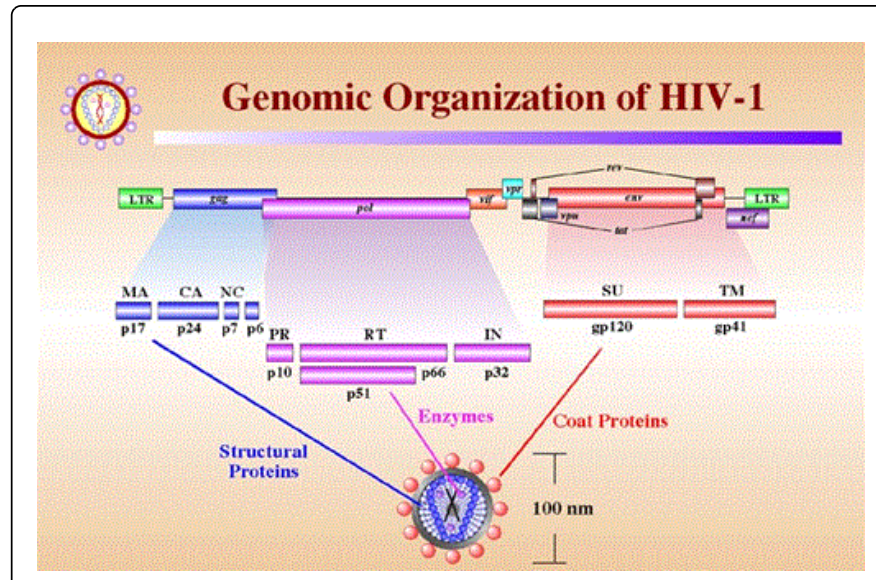

Figure 2: HIV genomic organization.

Among the regulatory proteins, Tat acts as a transcription factor and Nef down-regulates the CD4 expression of the host cell. Rev protein binds with rev responsive element (RRE) and aids in nuclear export and RNA splicing of HIV mRNA. Among the three accessory proteins of HIV, Vif actively take part in viral infectivity and host pathogen interactions, Vpu help in the releases of new viral particles from host cell and $\mathrm{Vpr}$ destroy the CD4 molecules within the endoplasmic reticulum (ER), helps in nuclear localization of viral preintegration complex and arrest host cell division at G2/M stage.

\section{Global Diversity and Prevalence of HIV}

HIV originates from zoonotic infection with SIV in West Africa. Due to error prone nature of HIV reverse transcriptase enzyme, the genomic structure of HIV is highly heterogeneous.

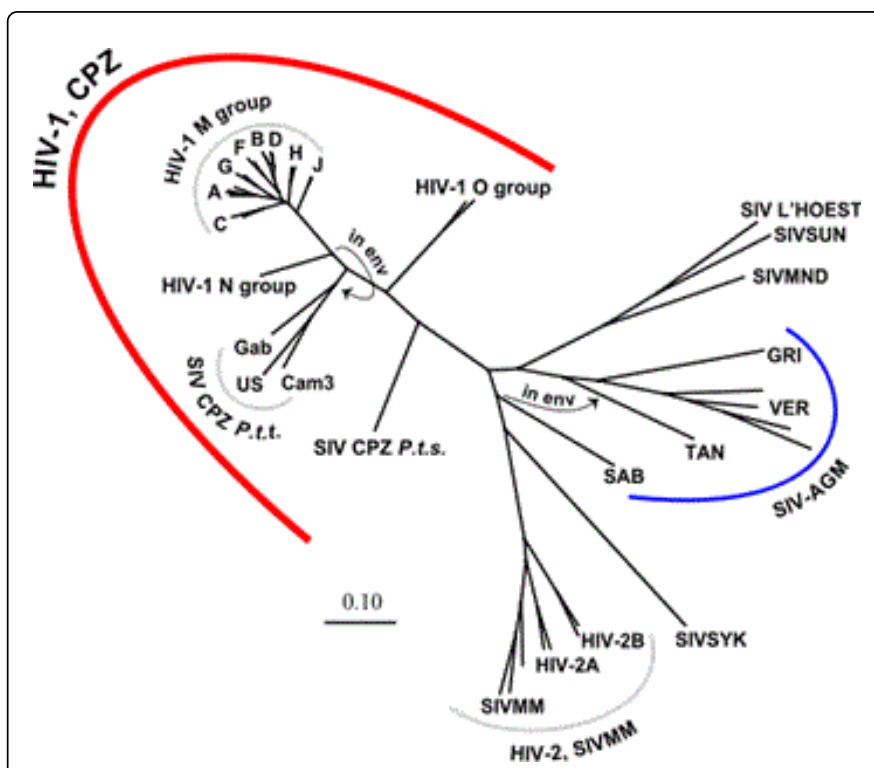

Figure 3: Phylogenetic tree of different clads of HIV.

Based on the sequence variation of the viral genome and its geographic distribution HIV is classified into several groups and subtypes. Apes were the origin of HIV-1 whereas HIV-2 originates 
from Sooty mengabey monkeys [11]. HIV-1 is classified into group $\mathrm{M}$, $\mathrm{N}, \mathrm{O}$ and $\mathrm{P}$. Group $\mathrm{M}, \mathrm{N}$ and $\mathrm{O}$ originates from Chimpanzee and Group P viruses originates from Gorillas. The distribution of HIV-2 and group N, O, P of HIV-1 is mainly confined in Central and West Africa. Group M HIV-1 is widely distributed throughout the world and is responsible for HIV pandemic. This group of HIV-1 is classified into nine pure subtypes based on their genetic organization and geographical distribution. The phylogenetic tree of different clads of HIV is described in Figure 3.

Other than the pure subtypes of HIV-1, a number of circulating recombinant forms of HIV-1 found in different parts of the worlds, which are originates from recombination of different subtypes. The different subtypes of HIV-1 differ with respect to their rate of evolution, response to antiretroviral therapy and development of development of drug resistance [12]. The global distribution of HIV-1 subtypes and circulating recombinants are described in Table 1.

\begin{tabular}{|l|l|}
\hline HIV-1 subtype/CRF & Global Distribution \\
\hline A & Russia, Democratic Republic of Congo, Tanzania. \\
\hline B & USA, Western European Countries, Japan, Australia. \\
\hline C & India, Brazil. \\
\hline D & Libya, Democratic Republic of Congo, Tanzania. \\
\hline F & West African Countries. \\
\hline G & Central Africa. \\
\hline H, J and K & Africa and Middle East Countries. \\
\hline CRF01-AE & South-East Asian Countries. \\
\hline CRF02-AG & Africa. \\
\hline CRF03-AB & Russia. \\
\hline CRF04-cpx & Greece, Cyprus. \\
\hline CRF05-DF & Democratic Republic of Congo. \\
\hline CRF06-cpx & Mali, Senegal, Nigeria. \\
\hline CRF07-BC & North West China. \\
\hline CRF08-BC & South East China. \\
\hline CRF09-cpx & Tanzania. \\
\hline CRF10-CD & Cameroon. \\
\hline CRF11-cpx & Senal, USA. \\
\hline
\end{tabular}

Table 1: Global distribution of HIV-1 subtypes and circulating recombinants.

As per UNAIDS report globally at present around 35.3 million people are living with HIV. With the advance of antiretroviral therapy and greater public awareness about HIV and AIDS, new spread of the virus reduced substantially. In the year 2012, globally 2.3 million new HIV infection reported compared to 3.4 million new cases reported in 2001. Although mother to child transmission of HIV decreased to a great extent by ART and incidences of heterosexual route of transmission also reduced by use of condoms, the rate of transmission of HIV among men who have sex with men and injecting drug users remain unaltered. The incidence of death among AIDS patients also decreased from 2.3 million in 2005 to 1.6 million in 2012. In India, around 2.1 million people are living with HIV. Similar to the global trends, the incidences of new infection decreased from 0.26 million in 2001 to 0.13 million in the year 2012. Opportunistic infections are one of the main hindrances in proper management of HIV related illness in the era of highly active antiretroviral therapy (HAART). Tuberculosis is the main opportunistic infection in India among the AIDS patients followed by HCV and HBV infection. In India around 1.7 million HIV patients currently required ART, of which only 0.57 million are availing the treatment [13].

\section{Life Cycle of HIV}

After infecting a new host the viral particles goes through the following stages to complete an entire life cycle: attachment, entry, reverse transcription, integration, transcription and translation, budding and release of new virions (Figure 4).

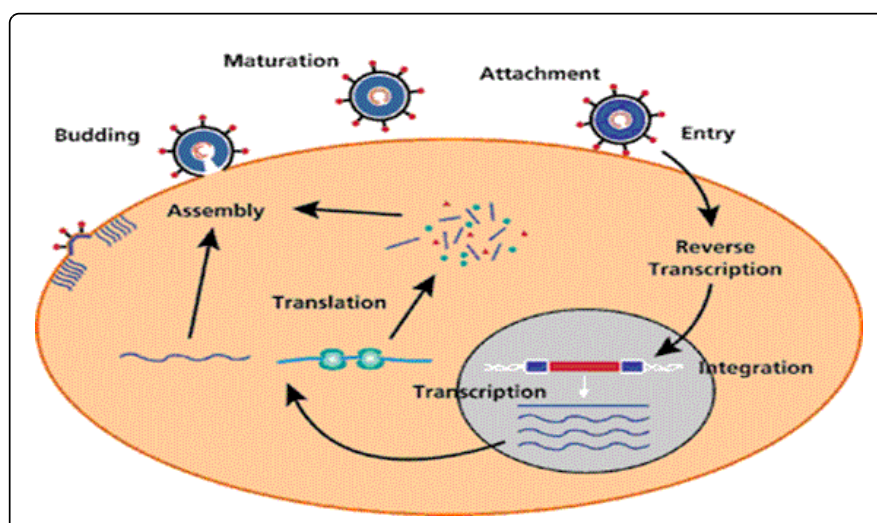

Figure 4: Life cycle of human immunodeficiency virus (HIV).

\section{Attachment}

After entering the host cells the gp120 anchored with the CD4 receptors of $\mathrm{T}$ helper cells. Although CD4 mediated attachment and entry is the main route of viral entry, CD4 independent viral entry into the host cell occurs in the astrocytes, renal epithelial cells and others $[14,15]$. The entry of HIV in this cells generate viral repository in brain and kidney respectively. Due to this in latter stages of the disease patients suffer from neuro cognitive disorders and nephropathy.

After attaching with CD4, gp120 interacts with chemokine coreceptors CCR5 or CXCR4. The selective use of these co-receptors called viral Tropism. The virions which uses CCR5 co-receptors are called M - Tropic and the viral strain is named R5 strains. Generally R5 strains appear in the early stages of the infection and interact with activated CD4 T helper cells and macrophages. So they have an active role in HIV transmission. They also aids in generation of viral repository in brain, lung and other body parts. The virions which use CXCR4 co-receptors are called $\mathrm{T}$ - Tropic and the viral strain is named $\mathrm{X} 4$ strains. They generally appear in the latter stage of the disease and interact with the resting $\mathrm{T}$ cells. They are responsible for rapid destruction of CD4 T cells and progression towards AIDS. Duel tropic viral strains also noticed which are able to use both the co-receptors. 


\section{Entry}

After attachment of the viral particle with host cell a conformational change take place in gp41, which guide entry of virion inside the cell. This is followed by un-coating of viral envelops and release of RNA.

\section{Reverse transcription}

In this stage of the viral life cycle, single stranded RNA is converted to double stranded DNA with the help of reverse transcriptase enzyme. Then assembly and nuclear export of pre integration complex (PIC) take place which have HIV double stranded DNA, matrix protein, integrase enzyme and accessory protein Vpr.

\section{Integration}

Here the double stranded DNA of HIV randomly integrated in the host chromosome with the help of integrase enzyme or stable circular double stranded DNA formed [16].

\section{Transcription and translation}

After generation of HIV proviral DNA, it uses the host cell machinery for transcription and translation of viral genome. During transcription 5' LRT of HIV genome acts as promoter. Host nuclear RNA pol II with the help of transcription factors synthesizes entire viral RNA. This RNA is exported from nucleus and packed in the new viral particles as their genetic material or spliced and translated to form long non functional viral polypeptides.

\section{Assembly and release}

The viral polypeptides are cleaved with the help of HIV protease and other host cell enzymes to give raise functional HIV proteins. Then they are assembled near plasma membrane of host cell and budding of the new virions take place. After that p6 nuclearcapsid protein of HIV interacts with the TGS101 and release new viruses in the blood [17].

\section{Monitoring Progression Of HIV Related Illness}

After initial detection of HIV in a patient, monitoring the progression of the disease status is important for overall quality of life of the patient. This also acts as an important guiding tool to determine the time of starting the antiretroviral therapy. CD4 T cell count and plasma HIV viral load are the two major tools used to monitor the disease progression. CD4 T cell counts guides clinicians to estimate the efficacy of the ART as well as the appearance of immunological failure status of a patient. Immunological failure is a condition where the CD4
$\mathrm{T}$ cell count of a patient on highly active anit-retroviral therapy (HAART) goes on decreasing with time $[18,19]$.

Monitoring the concentration of HIV virions in the plasma of an infected patient is considered as the gold standard for monitoring of the disease. Nucleic acid test (NAT) based HIV plasma viral load testing is widely used throughout the world. But implementation of this assay in routine use in resource limited settings with high prevalence rate of HIV like Sub Saharan Africa and South East Asia is difficult due to high cost of the test, sophisticated laboratory settings required to perform the test, requirement of high skilled technicians for interpretation of the results and transportation of samples from far interior regions to the reference laboratory [20,21]. In few settings EXAVIR v. 2 assay is used for HIV plasma concentration measurement. In this technology the reverse transcriptase enzyme activity of HIV is measured for counting viral particles and this test is around $80 \%$ cost effective then the NAT based viral load detection assay [22]. Other then these ELISA based p24 antigen concentration is also used as a surrogate marker for HIV viral load detection.

\section{Classification of HIV/AIDS Disease Stages}

In the year 2014, CDC revised the HIV/AIDS disease stages. As per the new guide line different stages of the disease classified into 5 groups with introduction of stage 0 followed by stage $1,2,3$ and unknown. Stage 0 is defined as a condition of a patient in which within 180 days of a confirmatory detection of HIV, another test shows negative or intermediate results. This occurs at the early stages of the acute infection. Disease stages 1 to 3 are classified based on the CD4 T cell count of the patient. In the absences of absolute CD4 T cell count, CD4 $\mathrm{T}$ cell percentage among the total lymphocyte count is used for disease staging. The details of this classification are described in Table 2. The different opportunistic infections and other complications related to HIV/AIDS are described in Table 3 [23].

\begin{tabular}{|c|c|c|c|c|c|c|}
\hline \multirow[t]{3}{*}{ Stage } & \multicolumn{6}{|c|}{ Age on date of CD4+ T-lymphocyte test } \\
\hline & \multicolumn{2}{|l|}{$<1$ years } & \multicolumn{2}{|c|}{$1-5$ years } & \multicolumn{2}{|l|}{$\geq 6$ years } \\
\hline & Cells/ $\mu \mathrm{l}$ & $\%$ & Cells $/ \mu l$ & $\%$ & Cells/ $/ \mu l$ & $\%$ \\
\hline 1 & $\geq 1,500$ & $\geq 34$ & $\geq 1,000$ & $\geq 30$ & $\geq 500$ & $\geq 26$ \\
\hline 2 & $750-1,499$ & $26-33$ & $500-999$ & $22-29$ & $200-499$ & $14-25$ \\
\hline 3 & $<750$ & $<26$ & $<500$ & $<22$ & $<200$ & $<14$ \\
\hline
\end{tabular}

Table 2: Classification of disease stages 1 to 3 based on the CD4 T cell count of the patent.

\begin{tabular}{|l|l|}
\hline Fungal Infections & Candidiasis, Coccidioidomycosis, Cryptococcosis, Histoplasmosi. \\
\hline Bacterial Infections & Tuberculosis, Mycobacterium avium complex, Salmonella septicemia. \\
\hline Viral Infections & Herpes simplex, Cytomegalovirus \\
\hline Parasitic Infections & Pneumocystis carinii pneumonia, Cryptosporidiosis, Toxoplasmosis. \\
\hline Malignances & Kaposi's sarcoma, Lymphoma, Invasive cervical cancer. \\
\hline Other Complications & Wasting syndrome due to HIV, Co-infection with HBV and HCV, Central and Peripheral nervous system disorders. \\
\hline
\end{tabular}

Table 3: Opportunistic infections and other complications related to HIV/AIDS. 
Citation: Acharya A, Vaniawala S, Parekh H, Misra RN, Wani M, et al. (2016) Human Immunodeficiency Virus: Discovery to Drug Resistance - A Review Update. Biol Syst Open Access 5: 154. doi:10.4172/2329-6577.1000154

Page 5 of 8

If any of the AIDS related opportunistic infections is detected in a patient, irrespective of its CD4 T cell count it is classified as stage 3 diseases. If there is no information available about CD4 $\mathrm{T}$ cell count or percentage, then it is classified as 'Unknown' stage [24].

World health organization (WHO) recommended HIV/ADIS disease staging based on the clinical findings of a confirmed HIV infected patient for the resource limited settings where facility of CD4 $\mathrm{T}$ cell counting is not available. The details of different disease stages are described in Table 4 [25].

\begin{tabular}{|c|c|}
\hline Clinical Stage & Clinical Conditions or Symptoms \\
\hline \multirow[t]{2}{*}{ Primary HIV Infection } & Asymptomatic \\
\hline & Acute retroviral syndrome \\
\hline \multirow[t]{2}{*}{ Clinical Stage 1} & Asymptomatic \\
\hline & Persistent generalized lymphadenopathy \\
\hline \multirow[t]{8}{*}{ Clinical Stage 2} & Moderate unexplained weight loss ( $<10 \%$ of presumed or measured body weight) \\
\hline & Recurrent respiratory infections (sinusitis, tonsillitis, otitis media, and pharyngitis) \\
\hline & Herpes zoster \\
\hline & Angular cheilitis \\
\hline & Recurrent oral ulceration \\
\hline & Papular pruritic eruptions \\
\hline & Seborrheic dermatitis \\
\hline & Fungal nail infections \\
\hline \multirow[t]{11}{*}{ Clinical Stage 3} & Unexplained severe weight loss (>10\% of presumed or measured body weight) \\
\hline & Unexplained chronic diarrhea for $>1$ month \\
\hline & Unexplained persistent fever for $>1$ month $\left(>37.6^{\circ} \mathrm{C}\right.$, intermittent or constant) \\
\hline & Persistent oral candidiasis (thrush) \\
\hline & Oral hairy leukoplakia \\
\hline & Pulmonary tuberculosis (current) \\
\hline & Severe presumed bacterial infections (e.g., pneumonia, empyema, pyomyositis, bone or joint infection, meningitis, bacteremia) \\
\hline & Acute necrotizing ulcerative stomatitis, gingivitis, or periodontitis \\
\hline & Unexplained anemia (hemoglobin $<8 \mathrm{~g} / \mathrm{dL}$ ) \\
\hline & Neutropenia (neutrophils $<500$ cells $/ \mu \mathrm{L}$ ) \\
\hline & Chronic thrombocytopenia (platelets $<50,000$ cells $/ \mu \mathrm{L}$ ) \\
\hline \multirow[t]{8}{*}{ Clinical Stage 4} & HIV wasting syndrome, as defined by the CDC (see Table 1, above) \\
\hline & Pneumocystis pneumonia \\
\hline & Recurrent severe bacterial pneumonia \\
\hline & Chronic herpes simplex infection (orolabial, genital, or anorectal site for >1 month or visceral herpes at any site) \\
\hline & Esophageal candidiasis (or candidiasis of trachea, bronchi, or lungs) \\
\hline & Extra pulmonary tuberculosis \\
\hline & Kaposi sarcoma \\
\hline & Cytomegalovirus infection (retinitis or infection of other organs) \\
\hline
\end{tabular}


Citation: Acharya A, Vaniawala S, Parekh H, Misra RN, Wani M, et al. (2016) Human Immunodeficiency Virus: Discovery to Drug Resistance - A

Page 6 of 8

\begin{tabular}{|l|}
\hline Central nervous system toxoplasmosis \\
\hline HIV encephalopathy \\
\hline Cryptococcus's, extra pulmonary (including meningitis) \\
\hline Disseminated nontuberculosis mycobacteria infection \\
\hline Progressive multifocal leukoencephalopathy \\
\hline Candida of the trachea, bronchi, or lungs \\
\hline Chronic cryptosporidiosis (with diarrhea) \\
\hline Chronic isosporiasis \\
\hline Disseminated mycosis (e.g., histoplasmosis, coccidioidomycosis, penicilliosis) \\
\hline Recurrent nontyphoidal Salmonella bacteremia \\
\hline Lymphoma (cerebral or B-cell non-Hodgkin) \\
\hline Invasive cervical carcinoma \\
\hline Atypical disseminated leishmaniasis \\
\hline Symptomatic HIV-associated nephropathy \\
\hline Symptomatic HIV-associated cardiomyopathy \\
\hline Reactivation of American trypanosomiasis (meningoencephalitis or myocarditis) \\
\hline
\end{tabular}

Table 4: HIV/AIDS disease staging as recommended by WHO.

\section{Antiretroviral Therapy}

At present 6 different classes of antiretroviral medicines are available in the market. As per current guidelines HIV patients cannot be treated with a single category of drug called monotherapy, which is one of the main reasons for emergences of drug resistance in HIV therapy. The recommended combination therapy of HIV consists of two or more different categories of drugs and called highly active antiretroviral therapy (HAART). After the introduction of HAART in the management of HIV, the rate of HIV/AIDS related mortality and morbidity reduced drastically. The different classes of US-FDA approved anti-retrovirals are as follows: Nucleoside Reverse Transcriptase Inhibitors (NRTIs), Non-Nucleoside Reverse Transcriptase Inhibitors (NNRTIs), Protease Inhibitors (PIs), Fusion Inhibitors, Entry Inhibitors and Integrase Inhibitors.

\section{Nucleoside reverse transcriptase inhibitors (NRTIs)}

NRTI is the first categories of antiretrovirals discovered for the treatment of HIV. They are prodrugs and remain inactive in-vitro. After entering inside human system with the help of cellular kinases inactive prodrugs phosphorylated and become active. Till date 7 NRTIs are approved by US-FDA. They are as follows: Zidovudine (azidothymidine; AZT or ZDV), Didanosine (ddI), Stavudine (d4T), Lamivudine (3TC), Abacavir (abacavir sulfate, ABC), Tenofovir disoproxil fumarate (tenofovir DF, TDF) and Emtricitabine (FTC).

NRTIs mimic the natural dNTPs in human cells and get incorporated in the elongating viral DNA chain. The NRTI molecules lack 3'-OH group or have some altered sugar moiety in their structure. So further elongation of the DNA chain blocks, as 3'-5' phosphodiester bond cannot be formed. This termination may take place during RNA dependent DNA synthesis or DNA dependent DNA synthesis by HIV reverse transcriptase.

HIV virions become resistance to NRTIs due to accumulation of mutations within the RT gene. Due to these mutations NRTIs cannot be incorporated in the elongating DNA chains, while the incorporation of natural dNTPs remains unaltered. So NRTIs no more able to stop the elongation of DNA and formation of new double stranded DNA continues [26,27]. M184V and associated mutations in RT gene follow these pathways of resistance towards NRTIs [28].

Another route of mechanism of resistance of NRTIs involves ATPdependent pyrophosphorolysis, where NRTIs is removed from the elongating DNA chain by HIV reverse transcriptase with the aid of ATP. So the elongation of the DNA chain resumes $[26,29]$. Thymidine analog mutations (TAMs) in the RT gene follow these pathways of resistance towards NRTIs [30].

With the accumulation of NRTI associated drug resistance mutations the activity of RT enzyme as well as the replicative capacity of HIV reduced substantially, which is compensated by appearance of secondary mutations.

\section{Non-nucleoside reverse transcriptase inhibitors (NNRTIs)}

NNRTIs are another class of HIV-1 reverse transcriptase inhibitors. They bind at the allosteric hydrophobic site of RT enzyme in the vicinity of polymerase active site of the enzyme. They are noncompetitive inhibitors of the enzyme. After binding with the HIV-1 RT, they bring a conformational change within the enzymatic active domain and block the enzyme-substrate interaction. So the RT enzyme loses its polymerization activity and elongation of new nucleic acid chain is terminated $[31,32]$. One of the unique feature of NNRTIs is 
they are only effective against HIV-1 reverse transcriptase. It is inactive against RT enzyme of all other lentiviruses [33]. Till date five NNRTIs are approved by US-FDA and they are: Nevirapine (NVP), Delavirdine (delavirdine mesylate, DLV), Efavirenz (EFV), Etravirine (ETR) and Rilpivirine (rilpivirine hydrochloride, RPV).

The side effect and toxicity index of NNRTIs are very high. Mutations within the NNRTI binding domains of the enzyme confer resistance to these drugs. All of the drugs are structurally similar and binds to the same NNRTI binding pocket of RT enzyme, so there is high level of cross-resistances between different NNRTIs. The genetic barrier of this group of drugs is very small as one or two mutations results in high level of resistance.

\section{Protease inhibitors (PIs)}

HIV-1 protease is an aspartic protease group of proteolytic enzyme. Their main function is to cleave the gag-pol polyproteins to generate functional HIV-1 structural proteins [34]. The protease inhibitors bind in the active catalytic domain of the enzyme and inhibit its functionality. They are competitive inhibitors of protease enzyme [35,36]. Till date eight PIs are approved by US-FDA, and they are: Saquinavir (saquinavir mesylate, SQV), Ritonavir (RTV), indinavir (indinavir sulfate, IDV), Nelfinavir (nelfinavir mesylate, NFV), Atazanavir (atazanavir sulfate, ATV), Fosamprenavir (fosamprenavir calcium, FOS-APV, FPV), Tipranavir (TPV) and Darunavir (darunavir ethanolate, DRV).

Due to similar structures of the PIs, they are high cross-resistances among them. Resistance to PIs appears due to loss of binding between the drug molecules and the enzyme, while the enzyme maintains its binding capacity with its natural substrates. Primary reasons for resistance of PIs are mutations in and around the enzymatic active domain of HIV-1 protease. Gradual accumulation of these mutations lowers the viral fitness which is compensated with the appearance of secondary mutations $[37,38]$.

\section{Integrase inhibitors}

$\mathrm{HIV}$ integrase enzyme is a $32 \mathrm{KDa}$ protein. It helps in the formation of pre integration complex, 3'- end processing of viral double strand DNA and strand transfer by formation of multimers [39]. Integrase inhibitors blocks the strand transfer reaction during integration of viral genome in the host chromosome, so they are also known as strand transfer inhibitors [40]. Till date three integrase inhibitors are approved by US-FDA, and they are: Raltegravir (raltegravir potassium, RAL), Dolutegravir (DTG) and Elvitegravir (EVG).

Mutations within the active domain of integrase enzyme results in resistance to integrase inhibitors. These mutations also reduces functional activity of the enzyme, so replicative capacity of the virus also compromised with the accumulation of integrase inhibitor mutations [41], which is compensated with the appearances of accessory mutations.

\section{Fusion inhibitors}

One of the essential part of HIV virus envelop fusion with host cell plasma membrane is interaction between two homo-polymeric domains of gp41 with host cell co-receptors. Fusion inhibitors are small peptide molecules which mimic one of the gp41 glycoprotein, so a heterologous protein-protein interaction takes place which prevents gp41 and host cell interaction and blocks viral fusion. The only one fusion inhibitor approved by US-FDA is enfuvirtide (T-20). Resistance to fusion inhibitor appears due to mutations in the C-terminal domain of gp41 glycoproteins [42].

\section{Entry inhibitors}

This class of drugs acts on the host cell co-receptors instead of HIV viral particle. They bind the trans-membrane domain of CCR5 coreceptors in their hydrophobic domains. This site of interaction between CCR5 and the drug molecule is different from the interaction domain of CCR 5 and gp120 of HIV virions. But binding of drug molecule with CCR5 imparts a conformational change in CCR5, which block it's binding with GP120 of HIV. So the viral particles cannot enter inside the host cell [43]. As the drug bind specifically with CCR5 co-receptors, it is only effective against the M5 viral strains of HIV-1. It is not able to block the entry of X4 HIV-1 strains. Maraviroc (MVC) is the only US-FDA approved CCR5 antagonists available in the market.

Resistance to Maraviroc appears due to tropism switch, interaction of virions with drug bounded CCR5 co-receptors and mutations in the v3 loop of HIV-1 gp120 [44-46]. Tropism switch appears in patients who harbor quasi-species of X4 strains during initiation of Maraviroc treatment. As the treatment progress, the population of M5 strains gradually reduces and the proportion of X4 strains increases, results in tropism switch [47].

\section{Conclusion}

It is evident that the HIV/AIDS challenge is long term in nature and hence requires robust and time tested solutions to address it. Therefore adequate research and development across the globe is an urgent global health priority for the current as well as coming generation. Although great strides have been taken in this regard in the past several decades, they are still not enough to effectively tackle the menace.

Of significance is the fact that as HIV/AIDS mainly affect low- and middle-income countries, the private sector might not have enough financial attractions and incentives to invest in long term research to develop and bring forth newer solutions to handle this disease.

\section{References}

1. Hymes KB, Cheung T, Greene JB, Prose NS, Marcus A, et al. (1981) Kaposi's sarcoma in homosexual men-a report of eight cases. Lancet 2: 598-600.

2. Centers for Disease Control (CDC) (1982) Pneumocystis carinii pneumonia among persons with hemophilia A. MMWR Morb Mortal Wkly Rep 31: 365-367.

3. Barré-Sinoussi F, Chermann JC, Rey F, Nugeyre MT, Chamaret S, et al. (1983) Isolation of a T-lymphotropic retrovirus from a patient at risk for acquired immune deficiency syndrome (AIDS). Science 220: 868-871.

4. Oleske J, Minnefor A, Cooper R Jr, Thomas K, dela Cruz A, et al. (1983) Immune deficiency syndrome in children. JAMA 249: 2345-2349.

5. Marx JL (1984) Strong new candidate for AIDS agent. Science 224: 475-477.

6. Marx JL (1985) A virus by any other name Science 227: 1449-1451.

7. Coffin J, Haase A, Levy JA, Montagnier L, Oroszlan S, et al. (1986) What to call the AIDS virus? Nature 321: 10.

8. Muesing MA, Smith DH, Cabradilla CD, Benton CV, Lasky LA, et al. (1985) Nucleic acid structure and expression of the human AIDS/ lymphadenopathy retrovirus. Nature 313: 450-458.

9. Dorfman T, Mammano F, Haseltine WA, Gottlinger HG (1994) Role of the matrix protein in the virion association of the human 
immunodeficiency virus type 1 envelope glycoprotein. J Virol 68 1689-1696.

10. Accola MA, Strack B, Gottlinger HG (2000) Efficient particle production by minimal gag constucts which retain the carboxyterminal domain of human immunodeficiency virus type 1 capsid-p2 and a late assembly domain. J Virol 74: 5395-5402.

11. Sharp PM, Hahn BH (2011) Origins of HIV and the AIDS pandemic. Cold Spring Harb Perspect Med 1: a006841.

12. Abecasis AB, Vandamme AM, Lemey P (2009) Quantifying differences in the tempo of human immunodeficiency virus type 1 subtype evolution. J Virol 83: 12917-12924.

13. UNAIDS (2013) "Report on the global AIDS epidemic".

14. Liu Y, Liu H, Kim BO, Gattone VH, Li J, et al. (2004) CD4-independent infection of astrocytes by human immunodeficiency virus type 1: requirement for the human mannose receptor. J Virol 78: 4120-4133.

15. Chen P, Chen BK, Mosoian A, Hays T, Ross MJ, et al. (2011) Virological synapses allow HIV-1 uptake and gene expression in renal tubular epithelial cells. J Am Soc Nephrol 22: 496-507.

16. Bushman FD, Fujiwara T, Craigie R (1990) Retroviral DNA integration directed by HIV integration protein in vitro. Science 249: 1555-1558.

17. Bieniasz PD (2006) Late budding domains and host proteins in enveloped virus release. Virology 344: 55-63.

18. Faal M, Naidoo N, Glencross DK, Venter WD, Osih R (2011) Providing immediate CD4 count results at HIV testing improves ART initiation. J Acquir Immune Defic Syndr 58: e54-59.

19. Jani IV, Sitoe NE, Alfai ER, Chongo PL, Quevedo JI et al., (2011) Effect of point-of-care $\mathrm{CD} 4$ cell count tests on retention of patients and rates of antiretroviral therapy initiation in primary health clinics: an observational cohort study. Lancet 378: 1572-1579.

20. Aldous JL, Haubrich RH (2009) Defining treatment failure in resourcerich settings. Curr Opin HIV AIDS 4: 459-466.

21. Usdin M, Guillerm M, Calmy A (2010) Patient needs and point-of-care requirements for HIV load testing in resource-limited settings. J Infect Dis 201 Suppl 1: S73-77.

22. Steegen K, Luchters S, De Cabooter N, Reynaerts J, Mandaliya K, et al. (2007) Evaluation of two commercially available alternatives for HIV-1 viral load testing in resource-limited settings. J Virol Methods 146: 178-187.

23. Jones JL, Hanson DL, Dworkin MS, Alderton DL, Fleming PL, et al. (1999) Surveillance for AIDS-defining opportunistic illnesses, 1992-1997. MMWR CDC Surveill Summ 48: 1-22.

24. Selik RM, Mokotoff ED, Branson B, Owen SM, Whitmore S et al., (2014) Revised surveillance case definition for HIV infection-United States. MMWR 63: 1-10.

25. World Health Organization (2007) WHO case definitions of HIV for surveillance and revised clinical staging and immunological classification of HIV-related disease in adults and children. World Health Organization, Switzerland.

26. Arion D, Kaushik N, McCormick S, Borkow G, Parniak MA (1998) Phenotypic mechanism of hiv-1 resistance to 3'-azido-3'-deoxythymidine (azt): Increased polymerization processivity and enhanced sensitivity to pyrophosphate of the mutant viral reverse transcriptase. Biochemistry 37: 15908-15917.

27. Meyer PR, Matsuura SE, So AG, Scott WA (1998) Unblocking of chainterminated primer by HIV-1 reverse transcriptase through a nucleotidedependent mechanism. Proc. Natl. Acad. Sci. USA 95: 13471-13476.

28. Shehu-Xhilaga M, Tachedjian G, Crowe SM, Kedzierska K (2005) Antiretroviral compounds: mechanisms underlying failure of HAART to eradicate HIV-1. Curr Med Chem 12: 1705-1719.

29. Boyer PL, Sarafianos SG, Arnold E, Hughes SH (2001) Selective excision of AZTMP by drug-resistant human immunodeficiency virus reverse transcriptase. J Virol 75: 4832-4842.
30. Naeger LK, Margot NA, Miller MD (2002) ATP-dependent removal of nucleoside reverse transcriptase inhibitors by human immunodeficiency virus type 1 reverse transcriptase. Antimicrob Agents Chemother 46: 2179-2184.

31. Spence RA, Kati WM, Anderson KS, Johnson KA (1995) Mechanism of inhibition of HIV-1 reverse transcriptase by nonnucleoside inhibitors. Science 267: 988-993.

32. Rittinger K, Divita G, Good RS (1995) Human immunodeficiency virus reverse transcriptase substrate-induced conformational changes and the mechanism of inhibition by nonnucleoside inhibitors. Proc Natl Acad Sci USA 92: 8046-8049.

33. Gao Y, Paxinos E, Galovich J, Troyer R, Baird H, et al., (2004) Characterization of a subtype D human immunodeficiency virus type 1 isolate that was obtained from an untreated individual and that is highly resistant to nonnucleoside reverse transcriptase inhibitors. J Virol 78: 5390-5401.

34. Kohl NE, Emini EA, Schleif WA, Davis LJ, Heimbach JC, et al. (1988) Active human immunodeficiency virus protease is required for viral infectivity. Proc Natl Acad Sci U S A 85: 4686-4690.

35. Huff JR, Kahn J (2001) Discovery and clinical development of HIV-1 protease inhibitors. Adv Protein Chem 56: 213-251.

36. Louis JM, Ishima R, Torchia DA, Weber IT (2007) HIV-1 protease: structure, dynamics, and inhibition. Adv Pharmacol 55: 261-298.

37. Chang MW, Torbett BE (2011) Accessory mutations maintain stability in drug-resistant HIV-1 protease. J Mol Biol 410: 756-760.

38. Menéndez-Arias L, Martínez MA, Quiñones-Mateu ME, MartinezPicado J (2003) Fitness variations and their impact on the evolution of antiretroviral drug resistance. Curr Drug Targets Infect Disord 3: 355-371.

39. Engelman A, Bushman FD, Craigie R (1993) Identification of discrete functional domains of HIV-1 integrase and their organization within an active multimeric complex. EMBO J 12: 3269-3275.

40. McColl DJ, Chen X (2010) Strand transfer inhibitors of HIV-1 integrase: bringing IN a new era of antiretroviral therapy. Antiviral Res 85: 101-118.

41. Hare S, Vos AM, Clayton RF, Thuring JW, Cummings MD, et al. (2010) Molecular mechanisms of retroviral integrase inhibition and the evolution of viral resistance. Proc Natl Acad Sci U S A 107: 20057-20062.

42. Lalezari JP, Henry K, O'Hearn M, Montaner JS, Piliero PJ, et al. (2003) Enfuvirtide, an HIV-1 fusion inhibitor, for drug-resistant HIV infection in North and South America. N Engl J Med 348: 2175-2185.

43. Kondru R, Zhang J, Ji C, Mirzadegan T, Rotstein D, et al. (2008) Molecular interactions of CCR5 with major classes of small-molecule anti-HIV CCR5 antagonists. Mol Pharmacol 73: 789-800.

44. Westby M, Smith-Burchnell C, Mori J, Lewis M, Mosley M et al. (2007) Reduced maximal inhibition in phenotypic susceptibility assays indicates that viral strains resistant to the CCR5 antagonist maraviroc utilize inhibitor-bound receptor for entry. 81: 2359-2371.

45. Yuan Y, Maeda Y, Terasawa H, Monde K, Harada S, et al. (2011) A combination of polymorphic mutations in V3 loop of HIV-1 gp120 can confer noncompetitive resistance to maraviroc. Virology 413: 293-299.

46. Berro R, Klasse PJ, Jakobsen MR, Gorry PR, Moore JP, et al. (2012) V3 determinants of HIV-1 escape from the CCR5 inhibitors Maraviroc and Vicriviroc. Virology 427: 158-165.

47. Westby M, Lewis M, Whitcomb J, Youle M, Pozniak AL, et al. (2006) Emergence of CXCR4-using human immunodeficiency virus type 1 (HIV-1) variants in a minority of HIV-1 infected patients following treatment with the CCR5 antagonist maraviroc is from a pretreatment CXCR4 using virus reservoir. J Virol 80: 4909-4920. 